\title{
Permanent dialysis access in a patient with a total artificial heart
}

\author{
Bryan B. Hair, BS, ${ }^{\mathrm{a}}$ Lee Kirksey, MD, ${ }^{\mathrm{b}}$ Michael Z. Tong, MD, ${ }^{\mathrm{c}}$ Edward Soltesz, $\mathrm{MD},{ }^{\mathrm{c}}$ and \\ Mazen Hanna, MD, ${ }^{\mathrm{d}}$ Cleveland, Ohio
}

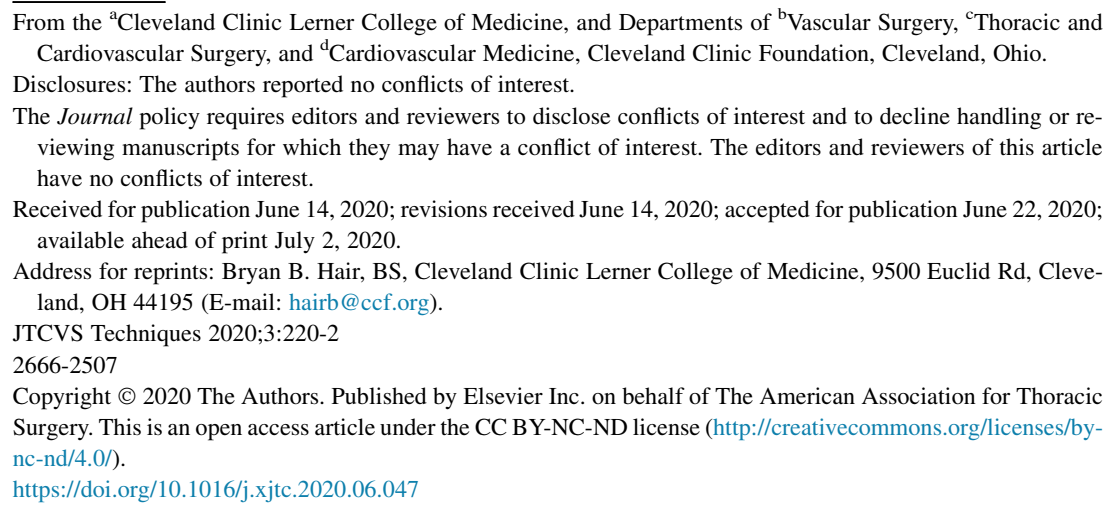

Video clip is available online.

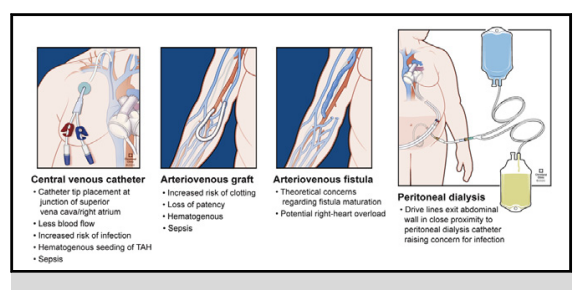

Dialysis access options and clinical considerations for patients with a TAH.

CENTRAL MESSAGE

The results of this case support that surgical fistula creation is a feasible option for patients with pulsatile mechanical circulatory support who require intermittent hemodialysis indefinitely.

See Commentaries on pages 223 and 225.
The total artificial heart (TAH) is a mode of mechanical circulatory support used for patients with severe biventricular heart failure. A primary risk of mechanical circulatory support implantation is acute kidney injury (AKI); unfortunately, patients with pre-existing renal dysfunction are at greater risk for postoperative AKI and may ultimately require intermittent hemodialysis (IHD). Herein, we report a seminal case in which a staged brachiobasilic arteriovenous fistula (bAVF) was successfully used until transplantation in a patient with a TAH.

\section{CASE}

The patient was a 48-year-old male, blood-type O, body mass index 38, with a long-standing history of nonischemic cardiomyopathy, including 2 previous sternotomies performed for mitral valve repair and mechanical aortic valve replacement, respectively. He progressed to end-stage heart failure with severe biventricular dysfunction and recurrent ventricular tachycardia prompting listing for heart transplantation. His condition worsened over the ensuing months, and given the severe biventricular dysfunction, ventricular tachycardia, and presence of a mechanical aortic valve, a TAH was implanted as a bridge to transplant with a preprocedural creatinine of $1.9 \mathrm{mg} / \mathrm{dL}$ (estimated glomerular filtration rate $38 \mathrm{~mL} / \mathrm{min} / \mathrm{m}^{2}$ ). It was felt that the moderate reduction in renal function was cardiorenal in nature, and that it would ultimately improve with mechanical support.

Postoperatively, the patient experienced severe vasoplegia, which resulted in oligoanuric AKI. IHD was initiated and, with minimal subsequent renal recovery, it was required indefinitely. Due to an anticipated long wait time (due to large body mass index, blood type $\mathrm{O}$, and significant allosensitization) he was discharged home on a freedom driver and listed for heart/kidney transplant as United Network for Organ Sharing status 1B. Although initially treated via tunneled dialysis catheter (TDC), after 6 months he suffered a catheter-related staphylococcal infection. This served as an impetus for discussions regarding alternative dialysis access options. Peritoneal dialysis was considered, but due to the proximity of the TAH drivelines to the potential peritoneal dialysis catheter site, this was not pursued. Surgical arteriovenous prosthetic graft placement would pose the risk of infection and was likewise deemed to be contraindicated (Figure 1).

Because of relatively lower infection risk, the decision was made to pursue a bAVF despite no experience per se with this approach in this context. For the initial hospitalization, the patient was already heparinized (as an 


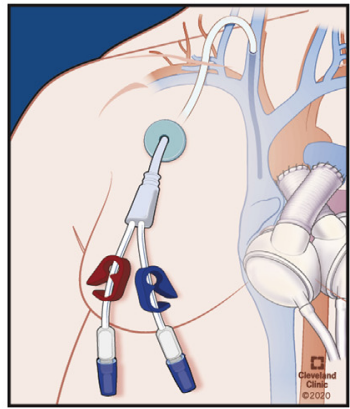

Central venous catheter

- Catheter tip placement at junction of superior vena cava/right atrium

- Less blood flow

- Increased risk of infection

- Hematogenous seeding of TAH

- Sepsis

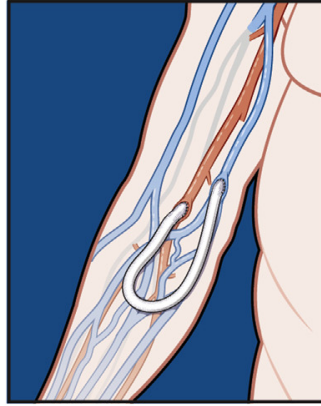

\section{Arteriovenous graft}

- Increased risk of clotting

- Loss of patency

- Hematogenous

- Sepsis

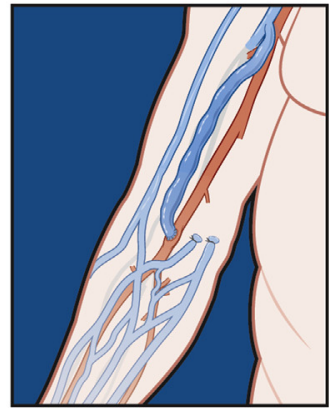

\section{Arteriovenous fistula}

- Theoretical concerns regarding fistula maturation

- Potential right-heart overload

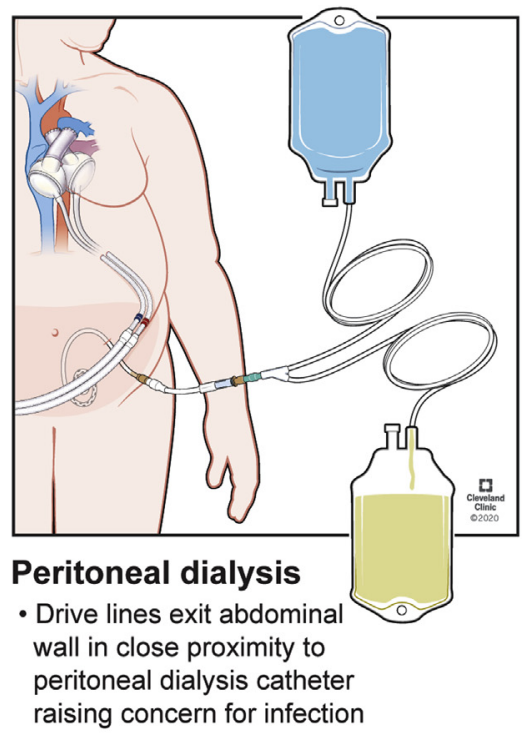

FIGURE 1. Dialysis access options and clinical considerations for patients with a TAH. TAH, Total artificial heart. Illustration created by David Schumick, BS, CMI. Reprinted with the permission of the Cleveland Clinic Center for Medical Art \& Photography, 2018.

inpatient) and the heparin was stopped on-call to the operating room. A wound drain was placed to reduce hematoma accumulation. Heparin was restarted 12 hours postoperatively at a flat rate, and then increased at 24 hours to a nomogram-driven dosing of activated partial thromboplastin time 55 to 65 seconds. Coumadin was given on the first postoperative evening to facilitate reanticoagulation. The patient was accompanied to the operating room by a certified advanced practice nurse with extensive training in mechanical support who remained with the patient throughout the procedure. The procedure was performed by a vascular surgeon within our Heart and Vascular Institute operating rooms. Per routine for all HVI procedures, a cardiothoracic anesthesiologist supervised the intraoperative delivery of anesthesia. A cardiac surgeon was immediately available if an unanticipated need were to arise.

Surgical bAVF was performed in a planned 2-stage approach at 6 and 9 months respectively post-TAH implantation. Postoperatively, an incisional hematoma was evacuated. Within 4 weeks, he tolerated consistent 2-needle cannulation of the bAVF and his TDC was promptly removed. Routine postoperative ultrasound demonstrated a conduit diameter of $10 \mathrm{~mm}$ with measured flow volumes of $1469 \mathrm{~mL} / \mathrm{min}$ (Figure 2). The bAVF functioned for 15 months, requiring 2 percutaneous interventions and one open revision for atypical aneurysmal degeneration of the arterial limb of the fistula until heart-kidney transplantation was accomplished. This study was completed with minimal risk, and informed consent was exempted by our institutional review board.

\section{DISCUSSION}

Following left ventricular assist device (LVAD) or TAH placement, AKI and the requirement of hemodialysis is a marker of poor prognosis. ${ }^{1,2}$ Studies suggest that the incidence of AKI following continuous-flow LVAD implantation lies between $7 \%$ and $45 \%$ whereas the incidence following TAH implantation is potentially even greater, between $19 \%$ and $64 \%{ }^{3,4}$ This is unsurprising, granted the hemodynamic instability and comorbidities characteristic of this population. Patients who fail to improve after optimizing hemodynamic parameters and volume status require renal-replacement therapy. ${ }^{4}$ For long-term hemodialysis, permanent dialysis access (arteriovenous fistula/graft placement) is preferred to avoid the high risk of infection

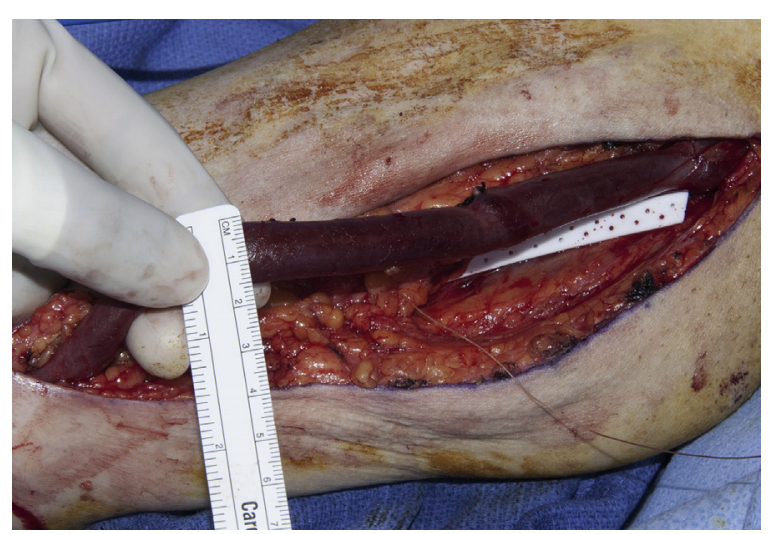

FIGURE 2. Intraoperative photograph of the patient's bAVF during the second stage of bAVF creation. 


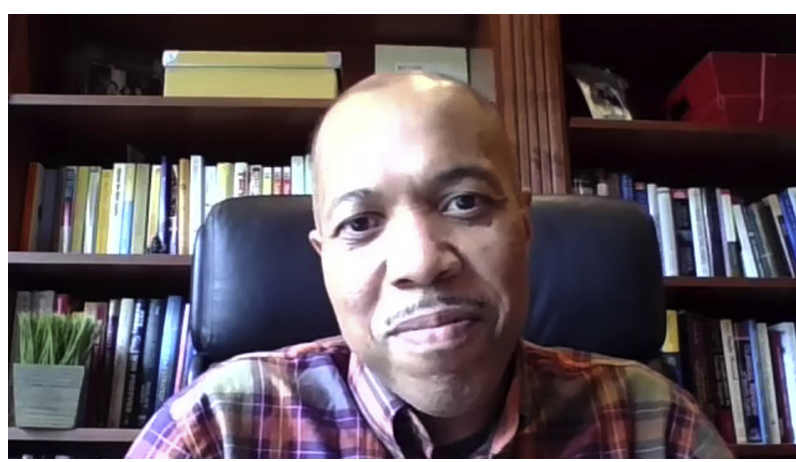

VIDEO 1. Discussion between a vascular surgeon and a heart failure/ transplant cardiologist regarding dialysis options for this patient with a total artificial heart (TAH). The rationale for pursuing an arteriovenous fistula is outlined in the context of potential risks and benefits. Video available at: https://www.jtcvs.org/article/S2666-2507(20)30336-9/fulltext.

associated with central venous catheters. ${ }^{3}$ Likewise, autologous fistula construction rather than prosthetic bridge grafting confers greater functional longevity and a lower risk of sepsis and hematogenous seeding of cardiac support hardware. ${ }^{5}$

Second- and third-generation LVADs use rotary pumps to produce continuous blood flow, and although cases of concomitant surgical bAVF have been described, there remains a theoretical concern of non-maturation. ${ }^{3}$ In contrast, the Food and Drug Administration-approved TAH produces pulsatile flow through a pneumatic driver to mitigate this challenge. ${ }^{2,4}$ In the case of our patient, after discussion regarding the various options for permanent access and the prompt need to remove the TDC, the vascular surgery team agreed that the technical aspects of fistula creation would not differ significantly (Video 1). It was acknowledged, however, that there was an inherent degree of uncertainty. One concern was the potential for super physiologic pulsatile flow to affect fistula arterialization and wall integrity. Despite this concern, the AVF did adequately mature with sufficient size, arterialization, wall thickening, and high flow rates. In our patient, it is unclear if the need for surgical revision of the non-cannulated anastomotic fistula segment was a result of the pulsatile flow produced by the TAH or unrelated.

As an increasing number of patients receive TAHs, it is inevitable that some will require IHD. Our case has demonstrated the feasibility of surgical fistula placement in a patient with a TAH with greater than 1 year of follow-up. Clinicians should view permanent dialysis access via AVF as an option that may be preferable to less-attractive alternatives. Future experience with this modality will further elucidate these risks and benefits.

\section{References}

1. Quader MA, Goodreau AM, Shah KB, Katlaps G, Cooke R, Smallfield MC, et al. Renal function recovery with total artificial heart support. ASAIO J. 2016;62: 87-91.

2. Copeland J, Arabia FA, Tenderich G, Marino A, Voigt O, Maiello C, et al. The total artificial heart. In: Mechanical Circulatory Support in End-Stage Heart Failure. Cham: Springer; 2017:317-30.

3. Patel AM, Adeseun GA, Ahmed I, Mitter N, Rame JE, Rudnick MR. Renal failure in patients with left ventricular assist devices. Clin J Am Soc Nephrol. 2013;8: 484-96.

4. Shah KB, Mankad AK, Tang DG, Kasirajan V. The total artificial heart. In: Eisen H, ed. Heart Failure: A Comprehensive Guide to Pathophysiology and Clinical Care. London: Springer London; 2017:695-704.

5. Santoro D, Benedetto F, Mondello P, Pipitò N, Barillà D, Spinelli F, et al. Vascular access for hemodialysis: current perspectives. Int J Nephrol Renovasc Dis. 2014;7: $284-5$. 